\title{
A CONSIDERATION UPON THE MOLECULAR MECHANISM OF MUSCULAR CONTRACTION AND RELAXATION
}

\author{
TORAO NAGAI, SHUNZO MARUYAMA AND MADOKA MAKINOSE* \\ Department of Physiology, Sapporo University of Medicine
}

For understanding the molecular mechanism of muscular contraction and relaxation in the actomyosin $(\mathrm{AM})$ system, it may be very useful to compare the phenomena found in the so-called "model fiber" with those in the AM solution, a more simple system than the former. Such a comparison was made by Szent-Györgyi and others $(1,2)$ between glycerinated muscle fiber and superprecipitation of AM solution. No referable experiment seems ever to have been made with regard to muscular relaxation.

The present authors attempted to compare the properties of glycerinated muscle fiber and those of the AM solution under a wide range of $\mathrm{KCl}$ concentration, with the purpose of assuming molecular changes in the AM system during muscular contraction and relaxation.

With the background of the recent progress of protein chemistry and its molecular mechanism, the problem is still very difficult so that only a partial solution can be expected.

\section{EXPERIMENTAL}

a) Glycerinated muscle fiber: Glycerinated muscle fiber was made from rabbit psoas muscle according to the method of Szent-Györgyi (3). The fiber was divided in the same diameter $(0.3 \mathrm{~mm}$.) and the same length $(30 \mathrm{~mm}$.) before the experiment.

b) Adenosinetriphosphate (ATP): ATP was prepared in our laboratory as $\mathrm{Ba}$-salt replacing $\mathrm{K}$-salt for use. This sample contained about half the amount of adenosinediphosphate (ADP) compared with ATP.

c) Measurement: The behavior of the glycerinated muscle fiber was observed under a wide range of $\mathrm{KCl}$ concentrations in the presence or absence of ATP in the following way.

i) Without load: The divided fibers were immersed in Petri glasses containing $\mathrm{KCl}$ solution in varied concentrations with or without ATP. The change of appearance was observed in the Petri glass. In the presence of ATP, the contracted fibers drew up on the glass plate 3 minutes after immersion, during which the contraction reached a stationary value. Under very careful treatment, the fibers were straightened without stretching. Then the length of the fibers

Received for publication, July 30, 1955.

* 永茾寊男 丸山俊蔵 牧之瀬 望 
was measured.

ii) With load: The divided fiber was placed in a glass cylinder as was shown in Maruyama's method (4), and was connected by an isotonic lever to a load of $200 \mathrm{mg}$. Thereafter, the glass cylinder was filled with various concentrations of $\mathrm{KCl}$ solution with or without ATP. The curve of contraction or relaxation was recorded on smoked paper on the kymographion.

\section{RESULTS}

1) Glycerinated muscle fiber and $\mathrm{KCl}$ concentration. The glycerinated muscle fiber changes its properties, particularly of elasticity and appearance, with the increase of $\mathrm{KCl}$ concentration (fig. 1, upper part). In physiological $\mathrm{KCl}$ concentration $(0.16 \mathrm{M})$, the fiber is opalescent and rigid.

When the $\mathrm{KCl}$ concentration increases, the transparency and elasticity of glycerinated muscle fiber increase gradually. Then the appearance of the fiber comes to bear a close resemblance to the living muscle. This phenomenon can be observed clearly about at $0.5 \mathrm{M} \mathrm{KCl}$ concentration. With a further increase of $\mathrm{KCl}$ concentration this tendency is intensified and at last some substance is dissolved out from the fiber. This substance may be concidered a protein of myosin or of the actomyosin group in reference to ordinary conditions in myosin-B preparation.

Under load, the significance of these phenomena becomes clearer (fig. 1, lower part). The extensibility of glycerinated muscle fiber rises with an increase of $\mathrm{KCl}$ concentration.
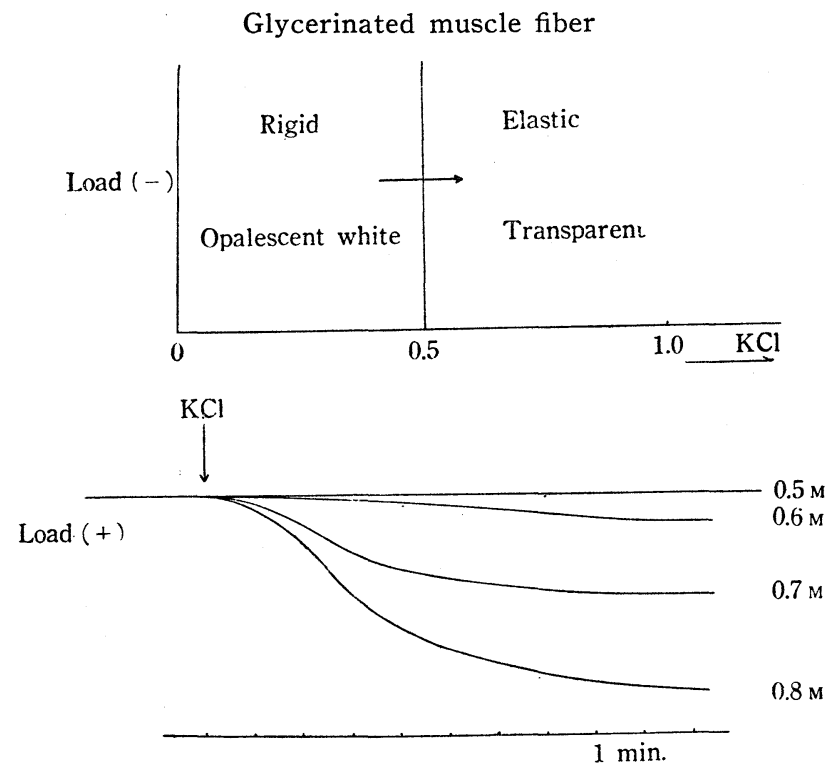

FIG. 1. Relation between the physical properties of glycerinated muscle fiber and $\mathrm{KCl}$ concentration.

Load : $200 \mathrm{mg}$. Temperature: $20^{\circ} \mathrm{C} . \quad p \mathrm{H}: 7.0$. 
This change of the properties of the model fiber can be induced by not only the change of $\mathrm{KCl}$ concentration but also by the addition of other agents, for example pyrophosphate etc. (cf. fig. 3).

That is to say, these changes of the properties of glycerinated muscle fiber are unspecific in regard to the sort of electrolyte.

It is important to observe this extension curve in great detail. At first, the fiber elongates, drawing an easy linear curve (first extension), and subsequently turns to steep extension (secont extension). In relatively low concentration of $\mathrm{KCl}$, the fiber usually shown only the first extension but in accordance with the increase of the $\mathrm{KCl}$ concentration, the duration of the first extension becomes shorter and at last the second steep extension appears with the start of elongation. During the first extension the fiber can practically recover its original length by unloading. That is to say this extension is almost an elastic deformation. But after the beginning of the second extension, the fiber cannot regain its original length by unloading even after an expectedly sufficient period. This means that the second extension includes plastic elongation.

That is to say, the extension of glycerinated muscle fiber by an increase of $\mathrm{KCl}$ concentration is in elastic extension at its beginning and as time goes on, it turns into plastic extension. When $\mathrm{KCl}$ concentration is high enough, the extension shows only plastic elongation from its beginning.

2) The influence of ATP. In the presence of ATP, the glycerinated muscle fiber shows maximum contraction within the limits of $0.5 \mathrm{M} \mathrm{KCl}$, corresponding to the extent in which the grade of rigor of the fiber is rather intense. Simultaneously with contraction by ATP, the fiber becomes elastic and transparent. When the concentration of $\mathrm{KCl}$ is over $0.5 \mathrm{M}$, the grade of shortening is suddenly decreased until one can observe no marked shortening (fig. 2). This phenomenon is almost identical to that formerly reported by A. G. Szent-Györgyi (5).
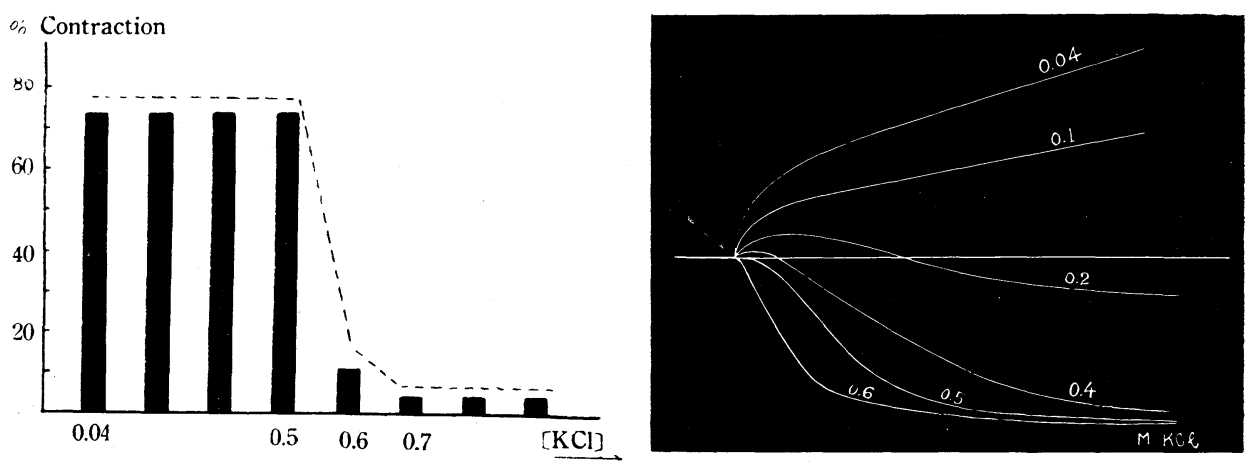

FIG. 2. The effect of ATP on glycerinated muscle fiber in relation to $\mathrm{KCl}$ concentration.

Temperature : $20^{\circ} \mathrm{C} . \quad p \mathrm{H}:$ 7.0. ATP : $0.2 \%$ ATP (with $10^{-3} \mathrm{M} \mathrm{MgCl}_{2}$ ).

The left: without load. The right: with load (200 mg.). 
Under load, the range of $\mathrm{KCl}$ concentration, in which the fiber shows contraction, becomes narrow. Then, at $0.5 \mathrm{M} \mathrm{KCl}$, the fiber shows no contraction, but, on the contrary, shows relaxation. This elongation is rather steep and this tendency is intensified by an increase of $\mathrm{KCl}$ concentration, like the case of elongation without ATP mentioned above. It is noteworthy that between two ranges of $\mathrm{KCl}$ concentration, in which the fiber shows only contraction or relaxation, there is another range in which the fiber shows contraction and relaxation successively. The extension in this case is usually relatively slow.

3) So-called "freeze up" phenomenon of the model fiber. Without ATP, the changes in the properties of the model fiber caused by an increase of the $\mathrm{KCl}$ (or the other agents) concentration are reversible within a certain range of the concentration of the applied agent. Namely with a decrease of $\mathrm{KCl}$ concentration the fiber returns to the rigid and opalescent white state again, and even under load the extensibility of the fiber disappears (fig. 3). With ATP, contracted fiber also gets into so-called "freeze up" state by replacing the ATP solution with the solution of low $\mathrm{KCl}$ concentration. It is considered that these two processes are caused by the same mechanism.

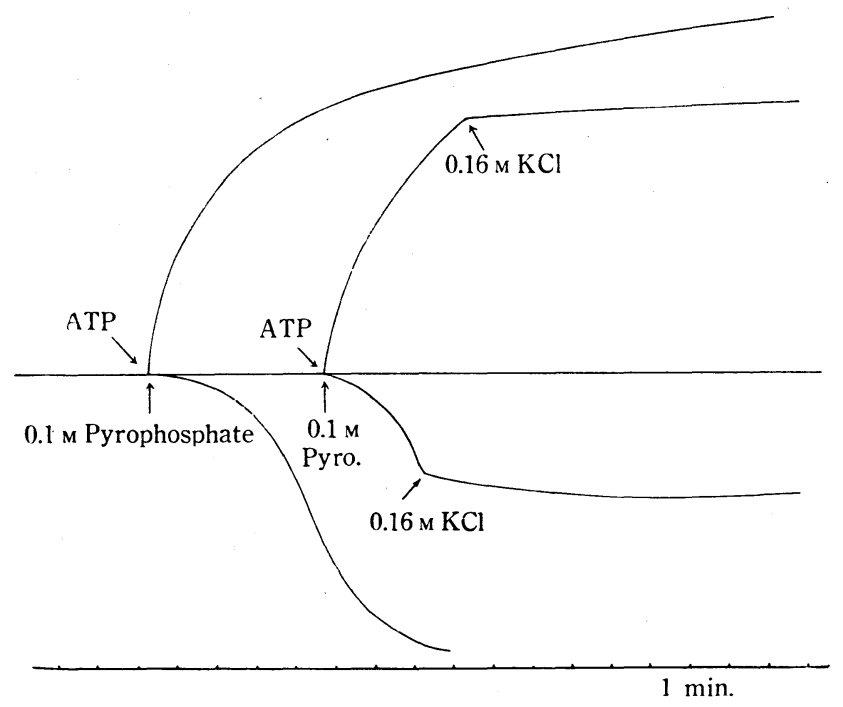

FIG. 3. So-called "freeze up" phenomenon of the model fiber by washing out of ATP with $0.16 \mathrm{M} \mathrm{KCl}$ solution.

ATP : $0.2 \% \mathrm{ATP}+10^{-3} \mathrm{M} \mathrm{MgCl}_{2}+0.16 \mathrm{M} \mathrm{KCl}$. Load : $200 \mathrm{mg}$. Temperature : $20^{\circ} \mathrm{C}$.

The glycerinated fiber can be elongated by pyrophosphate in the same way as with high concentration of $\mathrm{KCl}$.

4) Extension of glycerinated muscle fiber. The glycerinated muscle fiber contracted by ATP solution shows relaxation by the same agents which were 
used for the extension of uncontracted model fiber as shown in fig. 1 (fig. 4). This fact suggests that in these two extensions of the model fiber, the same molecular changes occur. fiber.

FIG. 4. Relaxation and derigor of the model

ATP : $0.1 \%$ ATP $+2 \times 10^{-3} \mathrm{M} \mathrm{MgCl}_{2}+0.16 \mathrm{M}$ $\mathrm{KCl}$. Load : $200 \mathrm{mg}$. Temperature : $20^{\circ} \mathrm{C}$.

$0.6 \mathrm{M} \mathrm{KCl}$ solution contains $2 \times 10^{-3} \mathrm{M}$ of $\mathrm{MgCl}_{2}$.

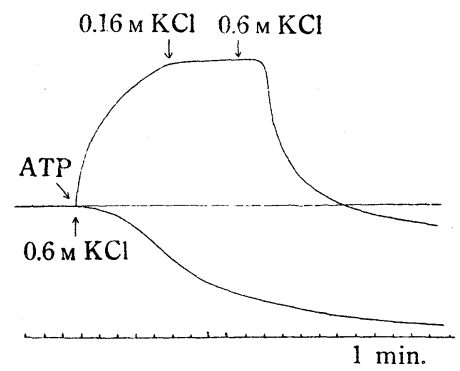

DISCUSSION

1) The correspondence between the behavior of AM solution and that of the model fiber

There are many reports about the behavior of $\mathrm{AM}$ solution at varied $\mathrm{KCl}$ concentration in the presence and absence of ATP $(1,2,6,7,8,9)$. In the absence of ATP, AM solution shows isoelectric precipitation within the range of low $\mathrm{KCl}$ concentration (below $0.4 \mathrm{M}$ ) and with an increase of $\mathrm{KCl}$ concentration the precipitate becomes gradually soluble and it appears as a clear and highly viscous solution. At last, in extremely high $\mathrm{KCl}$ concentration $(2 \mathrm{M})$ the high viscosity of the solution becomes very low. In the presence of ATP, superprecipitation, one of the most characteristic phenomena of the AM system, occurs at low $\mathrm{KCl}$ concentration and at high concentration of $\mathrm{KCl}$, ATP brings the high viscosity of AM solution to very low value, the same as observed at $2 \mathrm{M} \mathrm{KCl}$ in absence of ATP. This viscosity drop spontaneously recovers after a while.

The present authors have observed almost the same results as those reported by early investigators (10). These results were compared with the properties of the model fiber under varied $\mathrm{KCl}$ concentrations as shown in figs. 5 and 6 . In reference to $\mathrm{KCl}$ concentration, good correspondences between the behavior of the two materials can be observed either in the presence or in the absence of ATP.

In the absence of ATP, isoelectric precipitation of AM-solution corresponds to a rigid and opalescent state of the model fiber, which was named "rigor state" by Weber (11), and dissolution dissociation of the solution corresponds to the elastic and extensible state of the fiber, which has been called "derigor" by the present authors (12) in contradistinction to the term rigor used by Weber (fig. 5).

According to Szent-Györgyi $(1,2)$, the isoelectric precipitation is the aggregation of the AM molecule by intermolecular cohesive force as a result of minimum net charge of the molecule. This state is usually represented in present suthors' laboratory by the sign " $(\mathrm{AM})_{n}$."

Furthermore, Szent-Györgyi explained the dissolution of AM in high concentration of $\mathrm{KCl}$ as the dissociation of $\mathrm{AM}$ into actin (A) and myosin (M) caused by the increase of electrostatic repulsion between particles, and this repulsion 


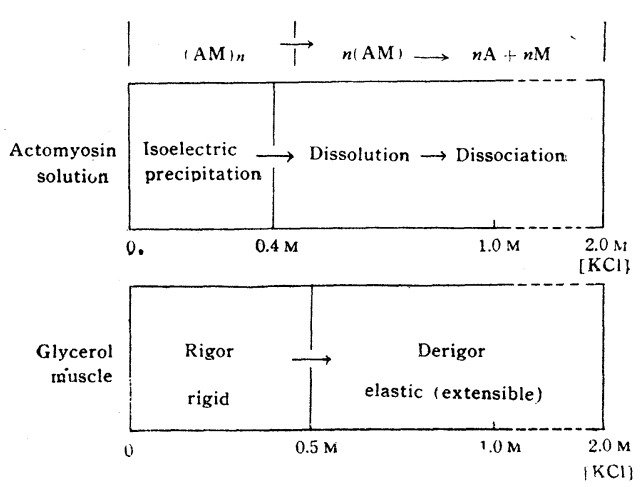

FIG. 5. Correspondence between the phases of AM solution and those of the model fiber, and molecular state corresponding to each of them.

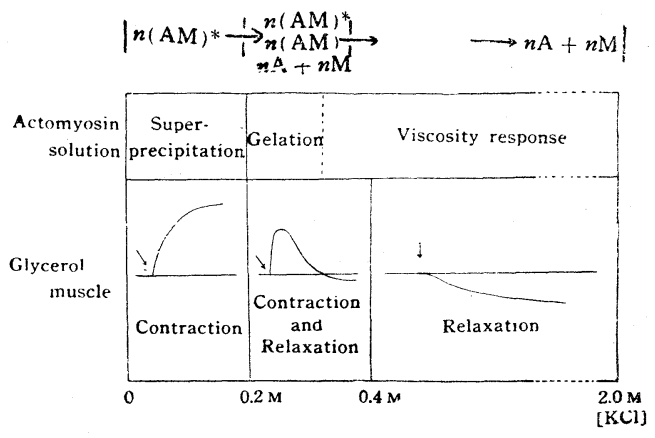

FIG. 6. The behavior of the model fiber in the presence of ATP and the molecular states corresponding to each phase.

is caused by an increase of negative charge by adsorption of $\mathrm{Cl}^{\prime}$ ion upon the particles. But, dissolution of AM does not usually mean the dissociation of AM molecule, and it may be considered that there occurs a loosening process of intermolecular linkage before the dissociation of AM into A and $\mathrm{M}$.

This is suggested by the presence of the AM molecule in the solution even when the $\mathrm{KCl}$ concentration increases over $0.5 \mathrm{M}$, because the high viscosity of $\mathrm{AM}$ is still kept in this $\mathrm{KCl}$ concentration and it is caused to decline by the addition of ATP. The state of AM (just descreibed) in which intermolecular linkage is weakend but the AM molecule is not dissociated, is represented by " $n(\mathrm{AM}) . "$

In company with the increase of $\mathrm{KCl}$ concentration, electrostatic repulsion of particles increases step by step. Then, at first, the disappearance of intermolecular linkage of AM takes place and, thereafter, by further increase of negative charge caused by greater increase of $\mathrm{KCl}$ concentration or addition of ATP, AM dissociates into A and M. This dissociated state is represented by "A + M."

The properties of the model fiber in rigor state suggest the presence of strong intermolecular linkage, and the behavior in derigor makes it easy for one to suppose a slackness of intermolecular linkage or dissociation of intramolecular bond of AM. Then it is reasonable to consider that rigor and derigor correspond respectively to isoelectric precipitation and to dissolution or dissociation. Also, it is possible to say that $(\mathrm{AM})_{n}$ corresponds to rigor and $n(\mathrm{AM})$ or $\mathrm{A}+\mathrm{M}$ corresponds to derigor as molecular mechanisms of each state of AM, according to the grade of negative charge. Moreover, it should be supposed that $n(\mathrm{AM})$ means an elastic elongation and $\mathrm{A}+\mathrm{M}$ a plastic one. This opinion of the present authors is just opposite to that of Szent-Györgyi's (13). He stated the view that plasticity of the model fiber is imputed to intermolecular dissociation of AM and elasticity to intramolecular dissociation of AM into A and $\mathrm{M}$. But it is difficult to accept the idea that the dissociation of AM which causes a molecular 
change higher than that brought about by dissociation of intermolecular linkage corresponds to the reversible elasticity, and that intermolecular dissociation of AM which causes weaker molecular changes corresponds to irreversible plastic properties.

In the above described experiments these molecular changes take place coincidentally. There are many transition forms between each of the two states. This is easily supposed from the obscurity of the boudaries between each of the phases of the AM system. Then it must be supposed that these three molecular states, viz. $(\mathrm{AM})_{n}, n(\mathrm{AM})$ and $\mathrm{A}+\mathrm{M}$, coexist at the same time, and that the statistical allocation of AM molecule in these states determines the property of any phase of AM system.

The same studies and attempts at systematization can be made upon the phenomena of AM system in the presence of ATP. As mentioned above, the correspondence between superprecipitation and contraction of the fiber was emphasized by Szent-Györgyi, and also he considered the dissociation of AM as the molecular mechanism of viscosity drop.

The present authors also consider that the molecular change of superprecipitation corresponds to that of muscular contraction (fig. 6). In the process of superprecipitation, it has been reported by Spicer (9) and Kasai (14), a member of the staff of our laboratory, that between isoelectric precipitation and syneresis there are at least two phases, viz., clear phase and aggregation. Furthermore, Kasai has reported on the mechanism of superprecipitation in detail. According to him, there are good reasons to suppose (1) that the clear phase is a kind of derigor state, (2) that the AM system premises moderate derigor before contraction and (3) that aggregation and syneresis (the AM molecule in these phases is represented by the sign $\left.(A M)^{*}\right)$ are due to the formation of a specific bond by the splitting energy of ATP.

These behaviors of the AM system suggest that the contracted AM system is in a moderate derigor state. These aspects are represented by the sign $n(\mathrm{AM})^{*}$.

Relaxation is considered to correspond to $\mathrm{A}+\mathrm{M}$ but this is a serious case. In this case the state of $n(\mathrm{AM}) *$ does not transform into $\mathrm{A}+\mathrm{M}$ directly but passes through the state of $n(\mathrm{AM})$ like the process from rigor to derigor in the absence of ATP; also $\mathrm{A}+\mathrm{M}$ corresponds to plastic relaxation and $n(\mathrm{AM})$ to elastic. In reference to $\mathrm{KCl}$ concentration, between superprecipitation and viscosity drop another phase of AM solution, gelation, was reported by Spicer (15) and Kasai (14). The molecular state of gelation can be supposed to be a mixed condition of $n(\mathrm{AM})^{*}, n(\mathrm{AM})$ and $\mathrm{A}+\mathrm{M}$.

2) A consideration of the molecular mechanism of contraction and relaxation of the musrle

In fig. 7 was summarized every molecular state corresponding to each phase of muscle fiber in reaction on the basis of the above discussion. Derigor and relaxation belong in almost the same molecular change as that caused mainly by electrostatic repulsion between the protein particles. At last, these two processes reach the state $\mathrm{A}+\mathrm{M}$ after passing through $n(\mathrm{AM})$, and, as stated 


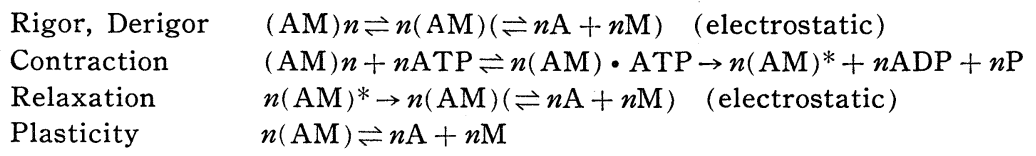

FIG. 7. The molecular states corresponding to each phase of the glycerinated muscle fiber.

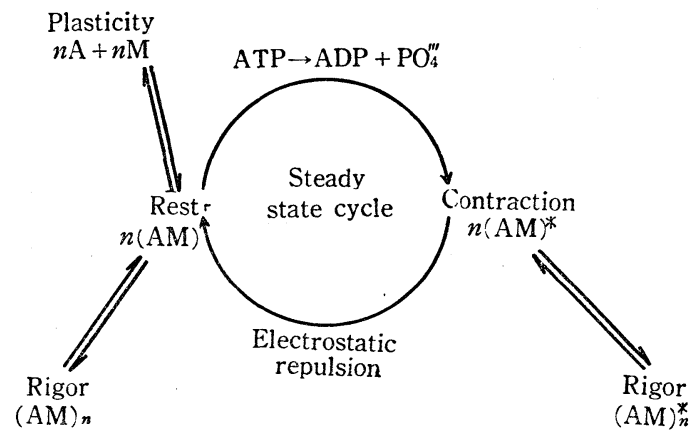

FIG. 8. The general relation between the physiological states of $\mathrm{AM}$ and its changes.

before, $n(\mathrm{AM})$ corresponds to elastic property of the model fiber and $\mathrm{A}+\mathrm{M}$ to the plastic one with, practically, many transition forms existing between them.

The relationship between these states is shown schematically in fig. 8, wherein the sign $(\mathrm{AM})_{n}^{*}$ represents the "freezed up" $\mathrm{AM}$ in the contracted state resulting from the washing out of ATP after contraction (cf. fig. 3). Much attention must be paid to the view (a) that in the physiological concentration of $\mathrm{KCl}$, the $\mathrm{AM}$ molecule in either model system, i.e., the glycerinated muscle or AM solution, is in the state of rigor and then the experiment should be started from this state, and (b) that in the process to contraction by ATP, the AM molecule must pass through the state of derigor.

In comparing the phenomena of living muscle and those of muscle model, great care must be exercised particularly with reference to these points.

About the molecular state of living muscle in resting state, no conclusive data has been gotten although many theories were offered by many investigators. For example, Szent-Györgyi $(1,2)$ supposed the dissociation of AM as the occasion for the prevention of contraction under the presence of ATP with the AM system, while Weber (11) considered the inhibition of ATP-splitting of AM. As shown in fig. 8, the present authors have supposed that the shortening or the tension development of the muscle fiber is performed by the revolution of the "steadystate cycle" composed of the contracting reaction resulted by splitting energy of ATP and of the relaxing reaction caused by unspecific ion activity (Nagai (16)). And they have also considered that the state of clear phase $(n(\mathrm{AM}))$ is a molecular state of resting muscle standing on the basis of the discussions presented above. This latter supposition is also suggested by the 
fact that the property of model fiber can be made to approach that of living muscle in resting state by means of moderate derigor.

However, in resting state the living muscle performs a so-called "resting metabolism" and shows a certain tension (resting tonus). In considering this point, as a molecular mechanism of resting muscle it may be adequate to presume the state of gelation in which the contracting reaction goes forward rather than the clear phase and, however, the contracting reaction is still less than the relaxing reaction. It is very interesting to compare the fact that by readding ATP, gelation turns into superprecipitation after passing through the clear phase (Spicer (15)) with the existence of latency relaxation preceding the contraction of the living muscle (17).

As mentioned above, the correspondence between gelation and the resting state of living muscle is very interesting physiologically. Thus, a number of discussions have been attempted upon the molecular mechanism of muscular contraction and relaxation. However, as stated in the introduction, there still exist some important and unsolved questions in this field.

For example, as was stated in Kasai's report (14), the present authors considered that muscular contraction is an active process caused by the formation of a specific bond by means of the splitting energy of ATP. But knowledge on the properties of this bond is still insufficient and, furthermore, the molecular kinetics of this process is entirely unknown. Recent investigators have had no effective method of making clear the mechanism of this mechano-chemical coupling. Thus, in the future, concentrated research is expected on this subject.

\section{SUMMARY}

1) Under a wide range of $\mathrm{KCl}$ concentration, the behavior of glycerinated muscle fiber was studied, and compared with that of the actomyosin solution.

2) Between the behaviors of the two materials, good correspondences were observed in reference to $\mathrm{KCl}$ concentration.

3 ) On the basis of 1) and 2), the molecular mechanism of muscular contraction and relaxation was discussed.

\section{REFERENCES}

1. Szent-GyörgYi, A. Chemistry of Muscular Contraction. New York: Academic Press, 1947.

2. Szent-GyöRgyi, A. Chemistry of Muscular Contraction. New York: Academic Press, 1951.

3. Szent-GyöRgyi, A. Biol. Bull. $96: 140,1949$.

4. MARUYAMA, S. Sap. Med. J. $4: 87,1953$.

5. SZENT-GYÖRGYI, A. G. Enzymologia $14: 246,1950$.

6. Needham, D. M., ET AL. J. Gen. Physiol. 27 : 355, 1944.

7. Mommaerts, W. F. H. M. J. Gen. Physiol. 31: 361, 1948.

8. CsÁpo, A. Acta Physiol. Scand. 19: 100, 1949.

9. SPICER, S. S. J. Biol. Chem. 199 : 289, 1952.

10. NAGAI, T. a) Sap. Med. J. $5: 154,1954$.

b) Seitai no Kagaku (Medical Science) 4: 194, 1953. 
11. Weber, H. H. eT AL. Advances in Protein Chemistry VII : 161, 1952.

12. NAGAi, T. And MiyazAKI, E. Sap. Med. J. $4: 232,1953$.

13. Szent-Györgyi, A. Chemical Physiology of Contraction in Body and Heart Muscle. New York: Academic Press, 1953.

14. KASAI, K. Sap. Med. J. in press.

15. Spicer, S. S. J. Biol. Chem. 190: 257, 1951.

16. NAGAI, T. Seitai no Kagaku (Medical Science) 6: 21, 1955.

17. Sandow, A. J. Cell. Comp. Physiol. 24 : 221, 1941. 